\title{
PREOPERATIVE TUMOR VOLUME IS ASSOCIATED WITH OUTCOME IN MALIGNANT PLEURAL MESOTHELIOMA
}

Harvey I. Pass, MD

Barbara K. Temeck, MD ${ }^{\mathrm{a}}$

Karen Kranda, RN ${ }^{\mathrm{a}}$

Seth M. Steinberg, $\mathrm{PhD}^{\mathrm{b}}$

Irwin R. Feuerstein, $\mathrm{MD}^{\mathrm{c}}$
Objectives: Our objective was to analyze the impact of preoperative and postresection solid tumor volumes on outcomes in 47 of 48 consecutive patients undergoing resection for malignant pleural mesothelioma who were treated prospectively and randomized to photodynamic therapy or no photodynamic therapy. Methods: From July 1993 to June 1996, 48 patients with malignant pleural mesothelioma had cytoreductive debulking to $5 \mathrm{~mm}$ or less residual tumor by extrapleural pneumonectomy $(n=25)$ or pleurectomy/decortication $(n=23)$. Three-dimensional computed tomographic reconstructions of preresection and postresection solid tumor were prospectively performed and the disease was staged postoperatively according to the new International Mesothelioma Interest Group staging. Results: Median survival for all patients is $\mathbf{1 4 . 4}$ months (extrapleural pneumonectomy, 11 months; pleurectomy/decortication, 22 months; $p_{2}=0.07$ ). Median survival for preoperative volume less than 100 was 22 months versus 11 months if more than $100 \mathrm{cc}, p_{2}=\mathbf{0 . 0 3}$. Median survival for postoperative volume less than 9 cc was 25 months versus 9 months if more than $9 \mathrm{cc}, p_{2}=$ 0.0002. Thirty-two of forty-seven $(68 \%)$ had positive $\mathrm{N} 1$ or $\mathrm{N} 2$ nodes. Tumor volumes associated with negative nodes were significantly smaller (median $51 \mathrm{cc}$ ) than those with positive nodes (median $166 \mathrm{cc}, p_{2}=0.01$ ). Progressively higher stage was associated with higher median preoperative volume: stage I, 4 cc; stage II, 94 cc; stage III, 143 cc; stage IV, 505 cc; $p_{2}=$ 0.007 for stage I versus II versus III versus IV. Patients with preoperative tumor volumes greater than $52 \mathrm{cc}$ had shorter progression-free intervals (8 months) than those 51 cc or less $\left(11\right.$ months; $\left.p_{2}=0.02\right)$. Conclusions: Preresection tumor volume is representative of $\mathbf{T}$ status in malignant pleural mesothelioma and can predict overall and progression-free survival, as well as postoperative stage. Large volumes are associated with nodal spread, and postresection residual tumor burden may predict outcome. (J Thorac Cardiovasc Surg 1998;115:310-8)
$\mathrm{M}$ alignant pleural mesothelioma (MPM) remains staging and management enigma to both medical and thoracic surgical oncologists. The poor

From the Thoracic Oncology Section, ${ }^{a}$ Biostatistics and Data Management Section, ${ }^{\mathrm{b}}$ National Cancer Institute, Diagnostic Radiology Department, ${ }^{\mathrm{c}}$ Warren Magnusen Clinical Center, National Institutes of Health, Bethesda, Md.

Read at the Seventy-seventh Annual Meeting of The American Association for Thoracic Surgery, Washington, D.C., May 4-7, 1997.

Received for publication May 7, 1997; revisions requested July 7 , 1997; revisions received Oct. 15, 1997; accepted for publication Oct. 16, 1997.

Address for reprints: Harvey I. Pass, MD, Harper Hospital, 3990 John R, Suite 2102, Detroit, MI 48201.

Copyright (C) 1998 by Mosby, Inc.

$0022-5223 / 98 \$ 5.00+0 \quad \mathbf{1 2 / 6 / 8 7 0 5 2}$ survival and persistent local recurrences after operative therapy have led to a feeling of nihilism among most thoracic surgeons, and no single agent or combination chemotherapeutic regimens exist with consistently higher partial response rates than $20 \%$ in human trials. ${ }^{1}$ Multimodality trials have been reported for MPM with reports of medians survivals of 22 months; yet on closer scrutiny of these trials, one finds that these are a select group of patients for whom a "margin negative" resection can be accomplished, and most long-term survivors did not have regional nodal spread. ${ }^{2}$

To resolve situations of treatment-result heterogeneity for a given neoplasm, it is important that a consistent, uniformly adapted staging system be in place. Ideally, the staging system would not require 
invasive techniques to identify prognostically unfavorable subgroups. As described by Rusch, the five staging systems before the International Mesothelioma Interest Group (IMIG) Staging System have "(been) to some extent imprecise and incompletely validated." 3 The IMIG staging system has only recently been available; at this writing it has only been validated in one series of 131 patients $^{4}$; and it has not been prospectively evaluated with regard to clinical versus operative stage.

Since 1993, a prospective randomized trial evaluating the role of intraoperative photodynamic therapy (PDT) in patients having operative cytoreduction of MPM and postoperative immunochemotherapy has been conducted at our institution. In 47 of the 48, T status has been assessed as solid tumor volume using a three-dimensional computerized tomographic reconstruction technique. Because no differences in survival or recurrence patterns were noted between the treatment arms in this completed study ${ }^{5}$ this study reports the prognostic variables associated with the patients who had uniform cytoreduction to $5 \mathrm{~mm}$ thickness of residual tumor independent of treatment group. Specifically, we were interested in the possible association of volumetrics with survival and recurrence and volumetric correlation with the new IMIG staging system as a whole, as well as individualized components such as nodal $(\mathrm{N})$ status.

\section{Methods}

Patients were considered for the prospective randomized trial of surgery, intraoperative PDT, and immunochemotherapy versus surgery and postoperative immunochemotherapy if, on review of their chest computerized tomogram before registration, it was believed that the disease was amenable to a subtotal extirpation such that the maximum thickness after debulking at any intrathoracic site was $5 \mathrm{~mm}$ or less. From July 1993 to June 1996, 63 patients were registered and randomized for a Phase III randomized trial investigating the use of intrapleural PDT for MPM, which was approved by the National Cancer Institute Institutional Review Board. There were 18 female and 45 male patients, all of whom signed informed consent. Of these, 15 patients ( 3 female and 12 male patients) were not considered for the volumetric analysis because of inability to debulk to the prerequisite $5 \mathrm{~mm}$ (eight patients, four PDT; four non-PDT), progression before operative intervention (six), or patient withdrawal before resection (one).

Either pleurectomy decortication alone or in combination with anatomic or nonanatomic resection and extrapleural pneumonectomy was performed, as long as the cytoreductive criteria to $5 \mathrm{~mm}$ could be met. This was, for most of the patients, a decision that was made at the time of exploration unless lung function, as defined preopera- tively with quantitative perfusion scanning, suggested that the affected lung was essentially nonfunctional, which implied that an extrapleural pneumonectomy would be required to meet cytoreductive criteria. The extrapleural pneumonectomy could be modified to include or not include portions of the pericardium or diaphragm. All patients having cytoreduction also had mediastinal nodal dissection and recording of intraoperative descriptors, some of which were later (1994) defined by the new IMIG staging system.

All patients fulfilling criteria for cytoreduction received postoperative immunochemotherapy (CIT) consisting of two cycles of tamoxifen citrate (INN: tamoxifen), $20 \mathrm{mg}$ orally bid given every day for 35 days, interferon- $\alpha 2 \mathrm{~B}$ ( 5 $\mathrm{mU} / \mathrm{m}^{2}$ ) delivered three times a week by subcutaneous injection, and cisplatin (CDDP), $25 \mathrm{mg} / \mathrm{m}^{2}$, delivered on days $8,15,22$, and 29 at least 30 minutes after interferon delivery.

Preoperative randomization determined whether the patient would receive $(n=23)$ or not receive $(n=25)$ intraoperative PDT at the time of maximal cytoreduction. As previously reported, ${ }^{5}$ the following descriptors were not statistically significantly different between the two groups: age, symptoms, interval from symptoms/diagnosis to treatment, performance status, pulmonary function, tumor volume (preoperative or postoperative), type of operation performed, histologic findings, involved nodes, or postoperative IMIG stage. Moreover, median survival and time to recurrence was virtually identical in comparing both groups. Therefore the analysis of outcomes and verification of the IMIG staging system were considered for all patients $(n=47)$ having complete intraoperative staging data and preoperative volumetrics.

Preoperative and follow-up tumor volume determinations. The volume of solid tumor ( $\mathrm{T}$ status) was objectively quantitated using the Voxel Scope II three-dimensional Imaging Work Station (Picker International, Highland Heights, Ohio) in 47 of the 48 patients having cytoreduction. For each patient the tapes of the computerized tomographic examinations were loaded into the memory of the system, and serial images of each study were depicted on the screen. One individual (B.T.) outlined the borders of the solid tumor using a handcontrolled mouse, with discrimination of solid tumor from fluid based on differences in tissue density. The system then calculated the three-dimensional volume in cubic centimeters of total solid tumor burden or of any desired subset or fraction.

The volumetrics were performed before resection, before initiation of immunochemotherapy (pre CIT), at the completion of CIT, and on follow-up examinations (every 3 months).

To compare the Voxell volumetric method for estimating size with more standard oncologic measures of tumor size, tumor areas were calculated using the same computerized tomograms in 43 of the patients. For each patient's scan, the individual slices (30 to 40 slices per scan) were divided into four quadrants, and the perpendicular diameters of solid tumor for each quadrant were recorded. The total solid tumor area was then calculated as the cumulative summation of the products of these perpendicular diameters (i.e., the sum of 120 to 160 diameter products 


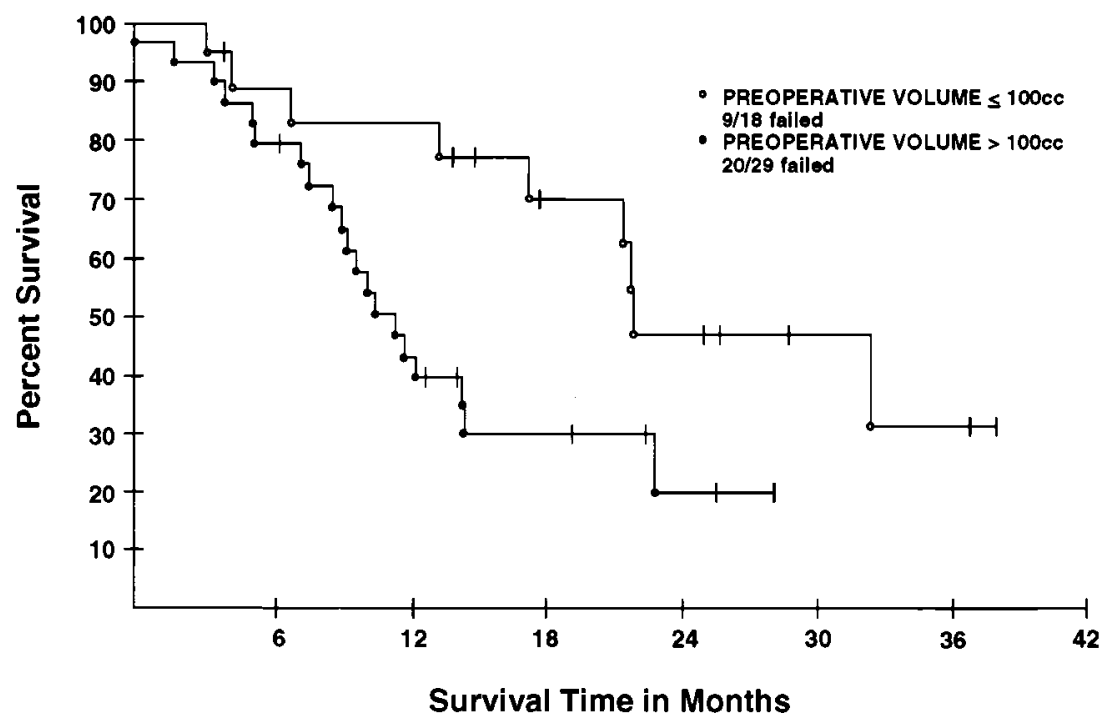

Fig. 1. Influence of preoperative size on overall survival of patients having cytoreduction for malignant pleural mesothelioma $\left(p_{2}=0.03\right)$

per patient) and correlated with the corresponding tumor volume for that patient.

Statistical methods. Survival time and time to progression in months were calculated from date of surgery until death, progression, or last follow-up as appropriate. The probability of survival or progression was calculated using the Kaplan-Meier method, ${ }^{6}$ and the significance of the difference between pairs of Kaplan-Meier curves was calculated using the Mantel-Haenszel procedure. ${ }^{7}$ The Cox proportional hazards model was used to identify which factors were jointly significant in the association with survival or recurrence. ${ }^{8}$ The factors considered for inclusion in univariate and Cox analyses were sex, histologic type of MPM, pretreatment platelet counts, type of operative procedure, nodal status, size (in cubic centimeters) before cytoreduction and pre CIT, and pathologic IMIG stage. Only those factors that were associated with at least a trend toward statistical significance in a univariate analysis $\left(p_{2}<0.20\right)$ were ultimately evaluated in a Cox model. Continuous parameters such as tumor size were divided into quartiles; because the trends in survival or recurrence-free survival were not necessarily linear over the four quartiles, for the Cox models, the data were dichotomized at the quartile that yielded the most significant result in the univariate analysis. In the case of preoperative tumor values, because a prior analysis had demonstrated that $100 \mathrm{cc}$ was a reasonable threshold, ${ }^{9}$ this threshold was also considered for evaluation in the Cox model in addition to a threshold based on a division at a quartile. In view of the limited number of patients in this study, these Cox models and the thresholds used in parameters evaluated in the Cox models were intended to be interpreted as hypothesis generating rather than definitive, with thresholds identified intended to be suggestive of effect rather than absolute. A backward selection algorithm was used to identify a reasonable model to represent the associations of var- ious parameters with outcome. The resulting model parameters $\left(b_{i}\right)$ were converted to relative risks by computing $\exp \left(b_{i}\right)$, where $\exp (a)=2.7183^{a}$. The $95 \%$ confidence interval for the relative risk was computed as $\left[\exp \left(b_{i L}\right)\right.$, exp $\left.\left(b_{i H}\right)\right]$, where $b_{i L}=b_{i}-1.96$ [estimated standard error $\left(b_{i}\right)$ ] and $b_{i H}=b_{i}+1.96$ [estimated standard error $\left.\left(b_{i}\right)\right] .{ }^{10}$ The relative risk indicates the risk associated with dying or progressing while being in a greater risk category compared with that of being in the lowest risk category. For example, a risk of 2.0 indicates twice the risk of dying during any interval if a patient has the trait associated with elevated risk compared with a patient without the trait. Jonckheere's test for trend ${ }^{11}$ was used to determine the significance of the association between stage and preoperative volume, whereas the Wilcoxon rank sum test compared sizes according to nodal status. All $p$ values are two-sided and are denoted by $p_{2}$.

\section{Results}

The median potential follow-up for the $47 \mathrm{pa}-$ tients was 23.1 months, with 29 of the 47 patients dead from mesothelioma as of the last analysis. The median survival for all patients was 14.4 months, and a trend for longer survival was observed in patients having pleurectomy/decortication compared with patients having extrapleural pneumonectomy (22 months vs 11 months, $\left.p_{2}=0.07\right)$. The mean $( \pm$ SEM $)$ preoperative volume for patients having a pleurectomy decortication was $88 \pm 16 \mathrm{cc}$ vs $418 \pm$ $86 \mathrm{cc}$ for extrapleural pneumonectomy (EPP) $\left(p_{2}<\right.$ $0.0001)$. No difference was observed in preoperative size $\left(p_{2}=0.70\right)$ in male patients $(n=32,267 \pm 64$ 


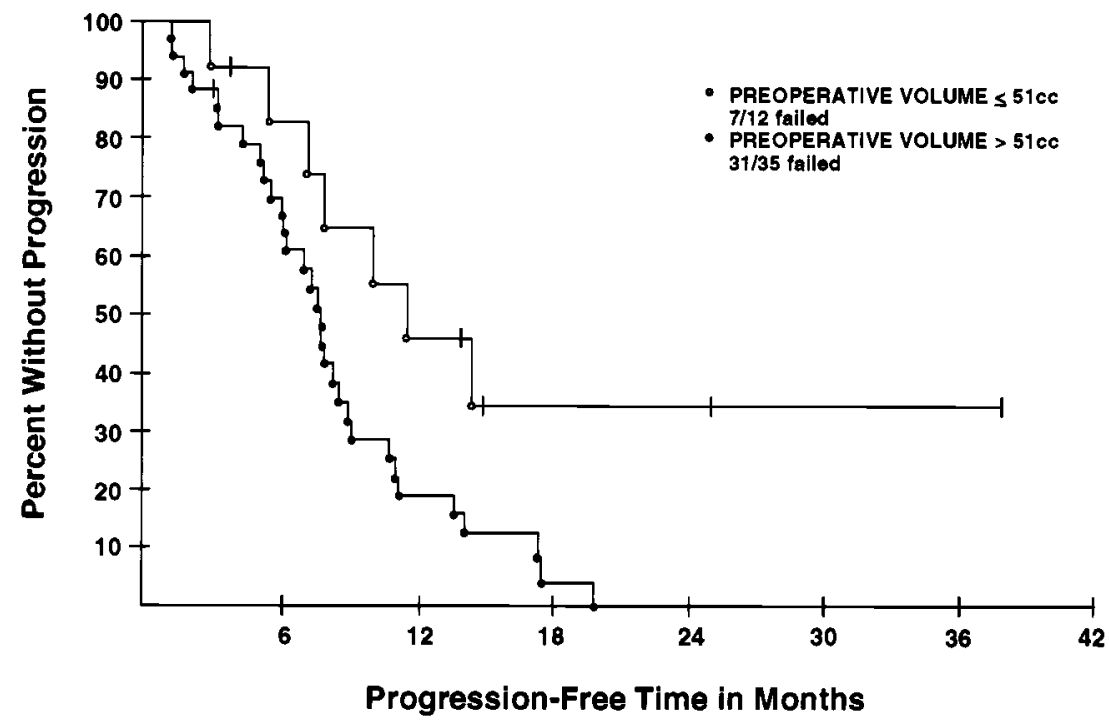

Fig. 2. Influence of preoperative size on progression-free survival of patients having cytoreduction for malignant pleural mesothelioma $\left(p_{2}=0.02\right)$

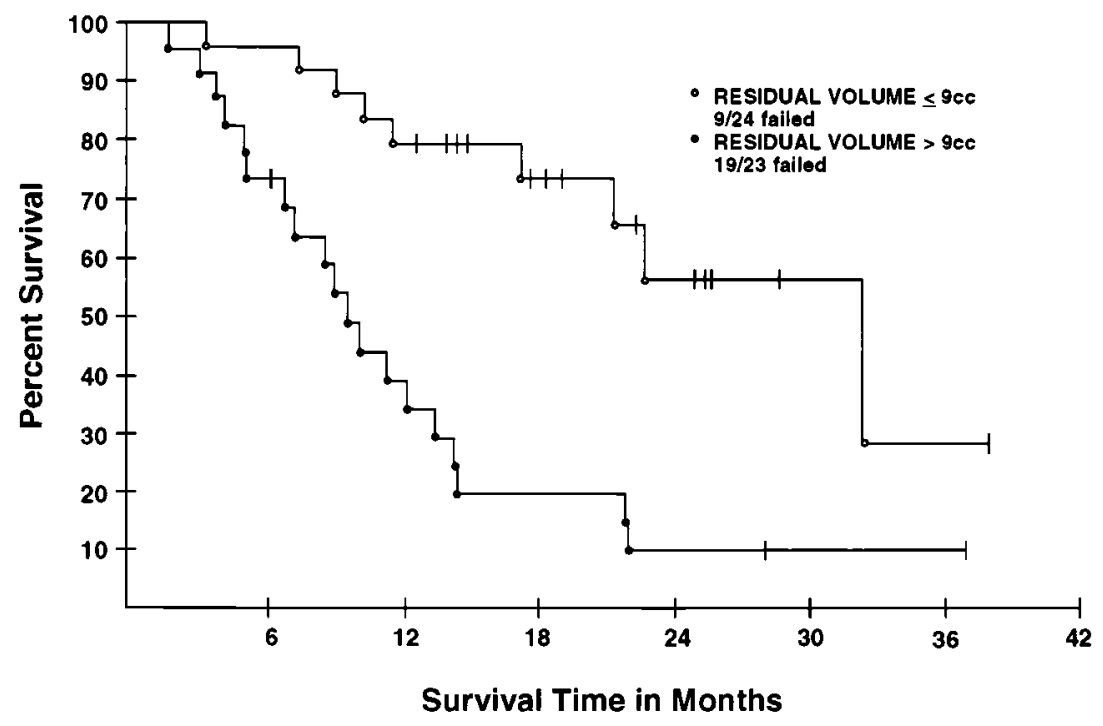

Fig. 3. Influence of residual tumor volume after cytoreduction on overall survival of patients having cytoreduction for malignant pleural mesothelioma $\left(p_{2}=0.0002\right)$

cc) compared with female patients $(n=15,257 \pm 92$ cc).

In univariate analyses, nodal status and cell type were not associated with survival $\left(p_{2}>0.20\right)$, but cell type was moderately associated with recurrencefree survival $\left(p_{2}=0.09\right)$, whereas nodal status was not associated with recurrence-free survival $(p=$ 0.48). In the Cox model, cell type was eliminated as not having prognostic importance for recurrencefree survival after including parameters that were statistically significant. It should also be noted that nodal status was considered universally (i.e., N1 and N2 status).

As seen in Fig. 1, a preoperative volume of $100 \mathrm{cc}$ or more was associated with a significantly worse prognosis compared with a preoperative volume less than $100 \mathrm{cc}$ ( 11 vs 22 months, $p_{2}=0.03$ ). Preoperative tumor volume also influenced the time to first recurrence, be it local or systemic (Fig. 2). No difference was observed between the postoperative, 


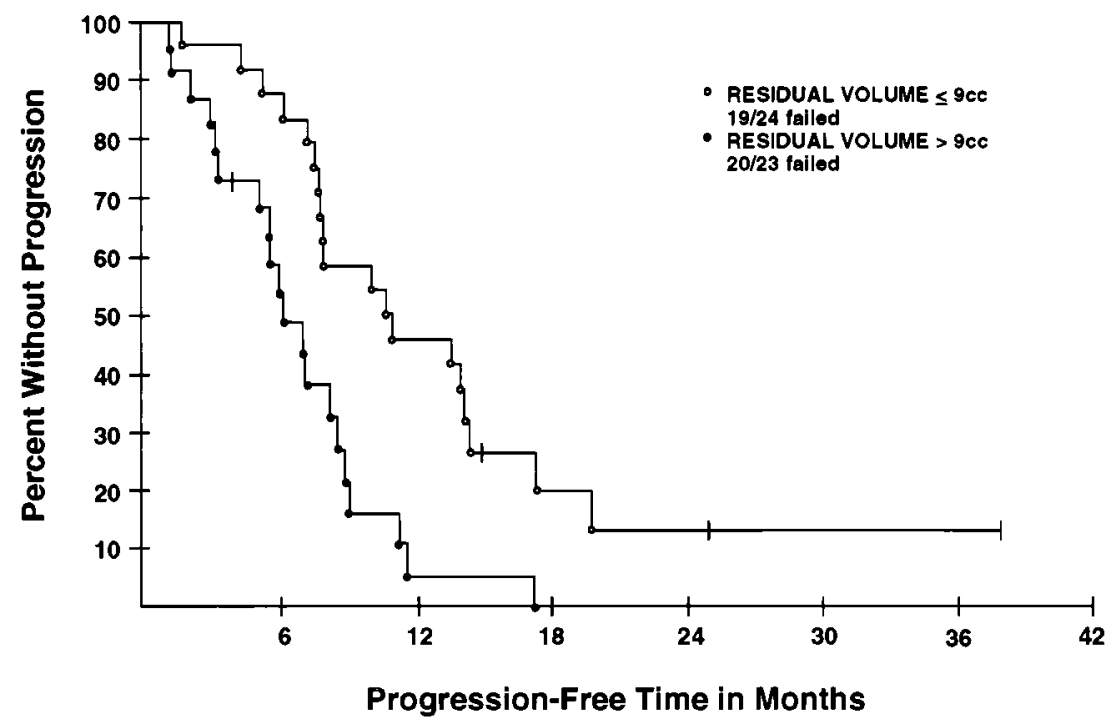

Fig. 4. Influence of residual tumor volume after cytoreduction on progression-free survival of patients having cytoreduction for malignant pleural mesothelioma $\left(p_{2}=0.001\right)$.

Table I. Correlation of preoperative tumor volume with lymph node status

\begin{tabular}{lccc}
\hline \multicolumn{1}{c}{ Node status* } & No. & $\begin{array}{c}\text { Median size } \\
(c c)\end{array}$ & $\begin{array}{c}\text { Wilcoxon } \\
\text { rank sum } P_{2}\end{array}$ \\
\hline N1 negative & 11 & 74 & 0.01 \\
N1 positive & 17 & 391 & \\
N2 negative & 18 & 70 & 0.04 \\
N2 positive & 29 & 163 & \\
No positive nodes & 15 & 51 & 0.009 \\
Any positive nodes & 32 & 166 & \\
\hline
\end{tabular}

*Only pneumonectomies $(n=25)$ and pleurectomy/lobectomy $(n=3)$ had N1 data; all 47 patients had mediastinal nodal dissections.

pre-CIT residual tumor volumes comparing patients who received a pleurectomy decortication or EPP (44 \pm 21 vs $\left.28 \pm 10 \mathrm{cc}, p_{2}=0.62\right)$. However, the volume of residual tumor, independent of the operation performed, influenced subsequent recurrence and survival. Patients left with a residual solid tumor volume (Fig. 3) of $9 \mathrm{cc}$ or more had a significantly decreased time to recurrence $\left(p_{2}=0.001\right)$ and a median survival (Fig. 4) of 9 months compared with 25 months for patients with less than $9 \mathrm{cc}$ residual volume $\left(p_{2}=0.0002\right)$.

Most of the 47 patients $(32,68 \%)$ in this series were found to have disease metastasized to the hilar (N1) or mediastinal (N2) nodes, and the tumor volumes for patients not having nodal metastases were significantly smaller than for those patients with $\mathrm{N} 1$ and/or N2 nodal metastases $(108 \pm 27$ vs $335 \pm 70 \mathrm{cc}, p_{2}=0.009$, respectively). Moreover, the presence or absence of either N1 or N2 disease was correlated with preoperative size (Table I).

All 48 patients who had cytoreduction were pathologically staged according to the IMIG staging system. There were four stage I patients, four stage II patients, 38 stage III patients, and two stage IV patients. Because of this lack of equal distribution of stages, a graded, stage by stage, decrease in survival was not seen. Fig. 5 illustrates, nevertheless, that stage grouping of I/II had a significantly better survival than stage grouping III/IV $\left(p_{2}=0.006\right)$. As depicted in Table II, the median preoperative sizes (i.e., T status) correlated well with the actual postoperative IMIG staging. There was a progressive, significant trend $\left(p_{2}=0.007\right)$ in tumor volume as postoperative IMIG stage increased from I to IV.

The results of multivariate analysis using the Cox model are depicted in Tables III and IV. Male sex, high platelet count, and large preoperative and postcytoreduction tumor volumes predicted decreased survival and increased chance for recurrence. A moderate correlation was found between platelet count and tumor volume $\left(r^{2}=0.53\right)$.

Because of the unavailability of the Voxell at all institutions that may need accurate tumor estimations, we attempted to validate the volumetrics using a more labor intense, classic method to estimate the 


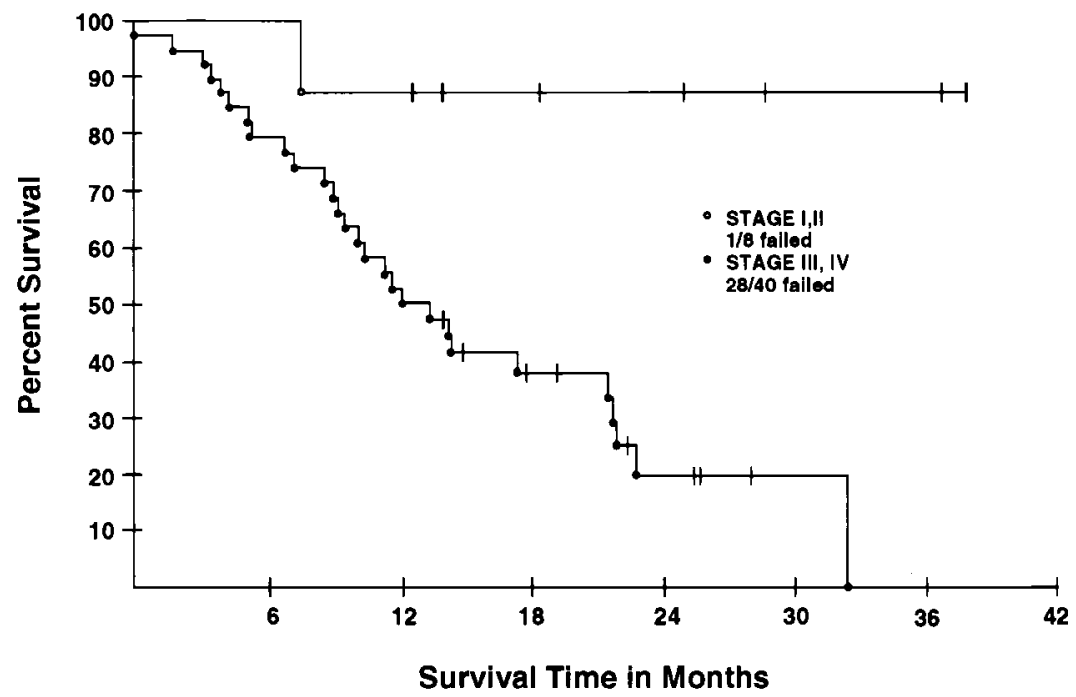

Fig. 5. IMIG stage after cytoreduction staging and survival of patients with malignant pleural mesothelioma $\left(p_{2}=0.006\right)$.

Table II. Association between preoperative volume and postoperative IMIG stage

\begin{tabular}{ccc}
\hline $\begin{array}{c}\text { Preoperative } \\
\text { size }(c c)\end{array}$ & $\begin{array}{c}\text { Postoperative } \\
\text { IMIG stage }\end{array}$ & $\begin{array}{c}\text { No. of } \\
\text { patients }\end{array}$ \\
\hline 4 & I & 3 \\
94 & II & 4 \\
143 & III & 38 \\
505 & IV & 2 \\
\hline
\end{tabular}

$p_{2}=0.007$ I vs II vs III vs IV by Jonckheere test.

area of solid tumor. Fig. 6 demonstrates that a 0.91 correlation existed between preoperative volume estimates and the sum of the products of the perpendicular diameters on the individual computerized tomographic slices for 43 patients.

\section{Discussion}

This study attempted to define whether T status in terms of computerized tomographic volumetric units was associated with (1) survival/recurrence for patients having cytoreductive surgery for MPM, (2) nodal involvement either intramediastinal or extramediastinal, or (3) postoperative IMIG stage. All data were prospectively entered on patients who participated in a single trial at our institution over a 3 -year period. We previously reported that the two groups were similar with regard to preoperative and postoperative volume measurements, and median survival and recurrence data did not differ between the groups, despite an additional therapy in one arm

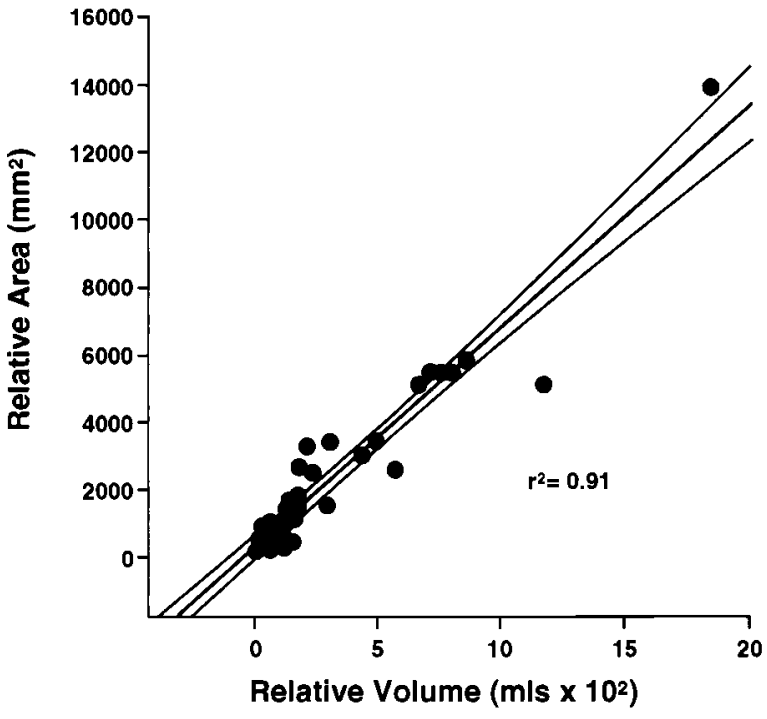

Fig. 6. Correlation of volumetric " $T$ " status with standard " $T$ " sizes as the sum of the products of the perpendicular diameters for each computerized tomographic slice (see text for details).

(PDT). ${ }^{5}$ We felt justified, therefore, in combining both groups as a single database to draw conclusions on the influence of T status in MPM.

It would seem obvious what the results of this study would be (i.e., the larger the size of the tumor, the more decreased the chance of long-term survival). The model on which this premise could be based 
Table III. Factors predictive of decreased survival by the Cox model

\begin{tabular}{lcccc}
\hline \multicolumn{1}{c}{ Variable } & $\begin{array}{c}\text { Parameter } \\
\text { estimate }\end{array}$ & $p$ Value & $\begin{array}{c}\text { Relative } \\
\text { risk }\end{array}$ & $\begin{array}{c}\text { 95\% Confidence intervals } \\
\text { for relative risk }\end{array}$ \\
\hline Male sex & 2.41 & 0.0001 & 11.09 & $(3.22,38.50)$ \\
Platelet count $>314,000$ & 1.12 & 0.0016 & 3.06 & $(1.24,7.59)$ \\
Preoperative volume $>100 \mathrm{cc}$ & 1.18 & 0.026 & 3.25 & $(1.15,9.20)$ \\
Postoperative volume $>9 \mathrm{cc}$ & 1.27 & 0.0042 & 3.55 & $(1.50,8.47)$ \\
\hline
\end{tabular}

Table IV. Factors predictive of recurrence by the Cox model

\begin{tabular}{lcccc}
\hline \multicolumn{1}{c}{ Variable } & $\begin{array}{c}\text { Parameter } \\
\text { estimate }\end{array}$ & $p$ Value & $\begin{array}{c}\text { Relative } \\
\text { risk }\end{array}$ & $\begin{array}{c}\text { 95\% Confidence intervals } \\
\text { for relative risk }\end{array}$ \\
\hline Male sex & 1.65 & 0.0004 & 5.21 & $(2.11,12.83)$ \\
Platelet count $>268,000$ & 1.27 & 0.0041 & 3.56 & $(1.50,8.44)$ \\
Preoperative volume $>52 \mathrm{cc}$ & 1.01 & 0.023 & 2.74 & $(1.16,6.50)$ \\
Postoperative volume $>9 \mathrm{cc}$ & 0.81 & 0.020 & 2.24 & $(1.13,4.46)$ \\
\hline
\end{tabular}

is lung cancer. A fundamental difference exists, however, between lung cancer and mesothelioma. Size can be well defined with lung cancer if the lesion is visible on plain chest $\mathrm{x}$-ray film or computerized tomogram without associated atelectasis or effusion. It becomes more difficult, despite advances in radiographic techniques, to apply more subjective qualifiers to lung cancer $T$ status (i.e., chest wall or mediastinal invasion). With mesothelioma, no techniques have been described that objectively define $\mathrm{T}$ status as a size, and all $\mathrm{T}$ status qualifications in the IMIG system are postoperative pathologic descriptors that put great weight on invasiveness: what is invaded, how deep or extensive is the invasion. Most thoracic surgeons are not going to be willing to provide a definitive answer to these descriptors in the mesothelioma patient, especially those with more bulk solid tumor than fluid, because they simply are not going to explore the patient for the resection of the disease. Therefore the more sobering and perhaps important portion of the data described here has to do with the predictive power of computerized tomographic volumetrics on lymph node status and overall stage of the disease.

Both N1 and N2 nodal tumor involvement could be correlated with increasing size of the tumor. To determine the exact cut off for size that would lead one to predict that the nodes are involved, a larger, prospective study is probably needed. However, it is curious that the cutoff size for involvement of the N2 nodes in this study is smaller than the cutoff size for involvement of the N1 nodes and probably reflects a reverse situation to that seen in most lung cancers. This would imply that the involvement of N1 nodes in MPM has a graver prognostic implication than the involvement of $\mathrm{N} 2$ nodes. For the 28 patients in our series who had N1 nodes recorded pathologically, a trend toward increased survival $\left(p_{2}=0.072\right)$ was observed if the nodes were free from tumor.

It is difficult to make definitive statements regarding substantiation of the new IMIG staging system for MPM from this study because a proclivity exists for higher stage patients registered. Nevertheless, the median survival of stage III disease of 11 months compares favorably with that published by Rusch and Venkatraman (11.5 months). ${ }^{4}$ Furthermore, it appears that the size of the tumor is predictive of the postoperative stage.

How can one explain the close correlation of $\mathrm{T}$ status with staging in this disease? One could speculate that unlike other thoracic malignancies, the biologic natural history of mesothelioma is one of local progression without metastases until late in the stage of the disease. This explains why certain investigators have commented on the fact that patients who have EPP will usually die from systemic rather than local disease. Therefore there seems to be a biologic size threshold that may be influenced by the local environment (i.e., stomal and matrix issues, growth factor elaboration) above which local factors can no longer prevent dissemination. This would imply that the biologic diversity of mesothelioma is more predictable (i.e., a smaller degree of differentiation heterogeneity resulting in a plethora of possible grades) than lung cancer.

Obviously other factors are involved with aggressiveness and predictability of mesothelioma other than size. One simply has to refer to the Cox model 
and see that sex and platelet number also independently contribute to outcomes. The Cox model clearly indicates that men are at a much greater risk of dying (relative risk of 11.09) or having a recurrence (relative risk $=5.21$ ) than women. The Cox model results indicate that both gender and size each contribute, independently and substantially, to the probability of dying or recurrence over time. In other words, regardless of gender, tumor size is important in prognosis, and, for any given tumor size, gender is important in prognosis.

These findings regarding the implications of size and prognosis in MPM need to be prospectively verified in larger groups of patients. It is reasonable to substitute a more quantitative "area" method to estimate size as described with the four-quadrant single-slice calculation, which correlated with our computed tomographic volumetrics. This will save cost but increase the time to calculate T status. Not only will such an endeavor possibly give more precise prognostic information without pathologic verification, but it may also be useful in delineating responses to nonsurgical (i.e., chemotherapy, immunotherapy, radiation, or gene therapy) treatments.

\section{REFERENCES}

1. Antman KH, Pass HI, Schiff PB. Benign and malignant mesothelioma. In: DeVita VT Jr., Hellman S, Rosenberg SA, editors. Cancer: principles and practice of oncology. 5th ed. Philadelphia: Lippincott-Raven; 1997. p. 1853-78.

2. Baldini EH, Recht A, Strauss GM, et al. Patterns of failure after trimodality therapy for malignant pleural mesothelioma. Ann Thorac Surg 1997;63:334-8.

3. Rusch VW. A proposed new international TNM staging system for malignant pleural mesothelioma. Chest 1995;108: 1122-9.

4. Rusch VW, Venkatraman E. The importance of surgical staging in the treatment of malignant pleural mesothelioma. J Thorac Cardiovasc Surg 1996;111:815-26.

5. Pass HI, Temeck BK, Kranda K, et al. A phase III randomized trial of surgery with or without intraoperative photodynamic therapy and postoperative immunochemotherapy for malignant pleural mesothelioma. Ann Surg Oncol. In press.

6. Kaplan EL, Meier P. Nonparametric estimation from incomplete observation. J Am Stat Assoc 1958;53:457-81.

7. Mantel N. Evaluation of survival data and two new rank order statistics arising in its consideration. Cancer Chem Rep 1966;50:163-70.

8. Cox D. Regression models and lifetables. J R Stat Soc 1972;34:187-202.

9. Pass HI, Kranda K, Temeck B, Feuerstein IM, Steinberg SM. Surgically debulked malignant pleural mesothelioma: results and prognostic factors. Ann Surg Oncol 1997;4:215-22.

10. Matthews DE, Farewell VT. Using and understanding medical statistics. Basel: Karger; 1985. p. 148-58.

11. Jonckheere AR. A distribution-free K-sample test against ordered alternatives. Biometrika 1954;41:133-45.

\section{Discussion}

Dr. Larry R. Kaiser (Philadelphia, Pa.). Dr. Pass, first at the National Cancer Institute and now in Detroit, has been one of the real leaders in the search for new knowledge and understanding of malignant mesothelioma. We all owe him a sincere debt of gratitude. Several years ago when we began working with this tumor, it was Dr. Pass that we called on.

One of the real problems in assessing efficacy of any therapy in this patient population has been the difficulty in evaluating the extent of the disease. This tumor does not lend itself to standard imaging modalities, and therapeutic trials have suffered. Dr. Pass has just reported on a technique that should allow us to significantly improve our ability to assess preoperative stage and thus make it possible for us to better assess efficacy of therapeutic interventions. This is a major step forward. It behooves those centers interested in treating mesothelioma-and there are painfully few of those it seems-to avail themselves of the Voxell volumetric technique, although the more work-intensive calculation of tumor areas provides a reasonable approximation. The bottom line, however, is that preoperative tumor volume correlates well with overall survival and validates the "T" component as important in the staging of this disease.

I have several questions for Dr. Pass. Was the decision to proceed with EPP versus pleurectomy/decortication based solely on preoperative tumor size or was this a decision made at the time of operation once the findings were noted? If so, does this increased tumor size alone account for the better survival seen in the lesser resection group?

I was somewhat surprised by the high incidence of lymph node involvement. Do you attribute this to the performance of a mediastinal lymph node dissection in all cases? Was mediastinoscopy performed? Would you have proceeded with EPP if mediastinoscopy had been positive? Was there any perioperative mortality in the EPP group? Your observations, perhaps, should make us rethink our approach to EPP, although you have also told us that the less tumor that remains postoperatively, the better the survival.

I am also a little bit confused about your postoperative measurements. If maximal debulking was carried out such that there was no gross residual disease, how was it possible to perform volume measurements or was the postoperative volume assessed at the time of operation? Where were the most common areas where tumor remained? Did you not take hemidiaphragm and pericardium in all pneumonectomy cases? Why, if less postoperative tumor correlates with improved survival, did all patients not have EPP? Finally, how would you propose that we make maximum use of the information that you have presented today?

Dr. Malcolm M. DeCamp (Boston, Mass.). Quantitating the tumor volume is a very difficult thing, and I think you have taken a very important step. We have been using magnetic resonance imaging scans to look at coronal, sagittal, and axial planes to evaluate areas of bulk disease. It helps us determine resectability and who may be a better candidate for pneumonectomy versus pleurectomy. I wonder whether your technique could be applied in the 
other tissue planes to give an even more accurate assessment of tumor bulk?

Dr. Pass. I would like to thank the discussants for their thoughtful questions.

The decision about EPP or pleurectomy was not made beforehand, but in the operating room. I would tell the patient that there is a chance that they may have an EPP and give them a percentage, but I let the findings at the operation dictate what I do.

The lymph node involvement is indeed different in our series. As opposed to other series that report 21-month median survivals, which are very carefully staged, we had $67 \%$ to $70 \%$ of our patients with positive nodes, which is very similar to what Valerie Rusch has reported in her LCSG experience, as well at Sloan-Kettering.

Considering now that I have done the curves and that there seems to be a trend that lymph node disease may be important in this, and Dr. Rusch has also shown that, I think that mediastinoscopy is not a bad idea in these patients considering that there will be therapies coming on-line that may be less toxic for possible induction therapies for these patients.
Sights of residual tumor are mainly on the diaphragm. The difference between my resections and other resections are that I do a "modified" EPP and that I do not commit the patient to the removal of the diaphragm. If I believe that I can get down to $5 \mathrm{~mm}$ by either going through the diaphragm or taking portions of the diaphragm, I do that, and then I will reconstruct the diaphragm to itself or with polytetrafluoroethylene. A positive margin is a positive margin wherever it is, and that is the way I have dictated my cases with mesothelioma.

In this particular series, the randomized trial, there was one mortality. So the total is 1 of 48 .

With regard to magnetic resonance imaging scans, I think magnetic resonance imaging scans are very, very useful, and I think magnetic resonance imaging scans may dictate whether you have to use invasive ways of staging, as Dr. Rusch has talked about, using either laparoscopy or thoracoscopy in looking under the diaphragm. Volume assessment is just going to give you a number and not comment on invasiveness or biologic characteristics. That is the one limitation of volume measurements.

\section{Bound volumes available to subscribers}

Bound volumes of The Journal of Thoracic and Cardiovascular Surgery are available to subscribers (only) for the 1998 issues from the Publisher, at a cost of $\$ 122.00$ for domestic, $\$ 151.94$ for Canadian, and $\$ 142.00$ for international subscribers for Vol. 115 (January-June) and Vol. 116 (July-December). Shipping charges are included. Each bound volume contains a subject and author index and all advertising is removed. Copies are shipped within 60 days after publication of the last issue of the volume. The binding is durable buckram with the Journal name, volume number, and year stamped in gold on the spine. Payment must accompany all orders. Contact Mosby, Inc., Subscription Services, 11830 Westline Industrial Drive, St. Louis, Missouri 63146-3318, USA; phone 800-453-4351 or 314-453-4351.

Subscriptions must be in force to qualify. Bound volumes are not available in place of a regular Journal subscription. 